\title{
The Normal Meromorphic Functions Family Concerning Higher Order Derivative and Shared Set by One-Way
}

\author{
Yi Li \\ School of Science, Southwest University of Science and Technology, Mianyang, China \\ E-mail: liyi@swust.edu.cn \\ Received October 7, 2011; revised October 28, 2011; accepted November 7, 2011
}

\begin{abstract}
Let $F$ be a meromorphic functions family on the unit disc $\Delta$, If for every $f \in F$ (the zeros of $f$ is a multiplicity of at least $k$ ) and if $f(z)=0$ then $\left|f^{(k)}(z)\right| \leq c$ and $\bar{E}_{f}^{(k)}(S) \subseteq \bar{E}_{f}(S) \quad(S=\{a, b\})$, then $F$ is normal on $\Delta$.
\end{abstract}

Keywords: Meromorphic Function, Normality Criterion, Shared Set by One-Way, Higher Order Derivative

\section{Introduction and Results}

First, we introduce the following definition:

Definition: For $a, b$ are two distinct complex values, we have set $S=\{a, b\}$ and

$$
\begin{aligned}
& \bar{E}_{f}(S)=\bar{E}_{f}(a, b) \\
& \quad=\{z:(f(z)-a))(f(z)-b)=0, z \in D\}
\end{aligned}
$$

If $\bar{E}_{f}(S)=\bar{E}_{g}(S)$, we call that $f$ and $g$ share $S$ in $D$; If $\bar{E}_{f}(S) \subseteq \bar{E}_{g}(S)$, we call that $f$ and $g$ share $S$ by One-way in $D$.

For the normal meromorphic functionsfamilyconcerning one-way sharing set, W. H. Zhang proved the following result [1]:

Theorem A. Let $F$ be a family of meromorphic functions in the unit disc $\Delta, a$ and $b$ be two distinct nonzero complex values. $S=\{a, b\}$, If for every $f \in F$, all of whose zeros is multiple, $\bar{E}_{f^{\prime}}(S)=\bar{E}_{f}(S)$, then $F$ is normal on $\Delta$.

W. H. Zhang continued considering the relation between normality and the shared set, and proved the next result [2]:

Theorem B. Let $F$ be meromorphic functions family in the unit disk $\Delta, a$ and $b$ be two distinct nonzero complex values. If for every $f \in F$, all of whose zeros is multiplicity $k+1$ at least ( $k$ is a positive integer), $\bar{E}_{f^{(k)}}(S)=\bar{E}_{f}(S)$, then $F$ is normal in $\Delta$.

In 2008, F. J. Lv got following theorem in [3]:

Theorem C. Let $F$ be a family of meromorphic function in the unit disk $\Delta, \mathrm{a}$ and $\mathrm{b}$ is two distinct nonzero complex values, $k$ is positive integer, $\Delta S=\{a, b\}$. If for every $f \in F$, all of whose zeros have multiplicity $k+1$ at least, $\bar{E}_{f^{(k)}}(S) \subseteq \bar{E}_{f}(S)$, then $F$ is normal in $\Delta$.

In this paper,we continue to discuss about normality theorem of meromorphic functions families concerning higher order derivative and shared set by one-way, and obtain main results as follow.

Theorem. Let $F$ be a meromorphic functions family on the unit disc $\Delta$, a and $\mathrm{b}$ is two distinct nonzero complex values, $k$ is positive integer, $S=\{a, b\}$, If for every $f \in F$, all of whose zeros have multiplicity $k$ at least, If

1) exists $c>0$, such that $f(z)=0 \Rightarrow\left|f^{(k)}(z)\right| \leq c$;

2) $\bar{E}_{f^{(k)}}(S) \subseteq \bar{E}_{f}(S)$;

then $F$ is normal in $\Delta$.

Remark: Through the following example, all of whose zeros have multiplicity $k$ at least is necessary.

Example: Let

$$
S=\{1, .-1\}, \omega_{1} \neq \omega_{2} \text { and } \omega_{i}^{k}=1, i=1,2 .
$$

a family of meromorphic function in the unit disk $\Delta$ be $F=\left\{f_{n}(z)\right\}$ there

$$
f_{n}(z)=n\left(\mathrm{e}^{\omega_{1} z}-\mathrm{e}^{\omega_{2} z}\right), n=1,2,3, \cdots .
$$

Obvously $f_{n}(z)=f_{n}^{(k)}(z)$ and 


$$
f_{n}^{(l)}(z)=n\left(\omega_{1}^{l} \mathrm{e}^{\omega_{1} z}-\omega_{1}^{l} \mathrm{e}^{\omega_{1} z}\right), \quad(l=1,2,3, \cdots, k-1),
$$

Hence $z=0$ satisfy 1) and 2) and all of whose zeros of $f_{n}(z)$ have multiplicity $k-1$ at most, Since

$$
f^{\#}(0)=\frac{\left|f^{\prime}(0)\right|}{1+|f(0)|^{2}}=n\left|\omega_{1}-\omega_{2}\right| \rightarrow \infty(n \rightarrow \infty),
$$

$F$ is not normal at $z=0$ by Marty Theorem.

\section{Lemmas}

Lemma 1 [4]. Let $F$ be meromorphic functions families in the unit disk $\Delta$, all of whose zeros have multiplicity $k$ at least. If for every $f \in F$, there exists $A>0$, such that $\left|f^{(k)}(z)\right| \leq A$ when eve $f(z)=0$. If $F$ is not normal in $\Delta$, then for every $0 \leq \alpha \leq k$, there exists $0<r<1,\left|z_{n}\right|<r, \quad f_{n} \in F, \rho_{n} \rightarrow 0$, such that $g_{n}(\zeta)=\frac{f_{n}\left(z_{n}+\rho_{n} \zeta\right)}{\rho_{n}^{\alpha}}$ converges locally and uniformly to a noncontant meromorphic function $g(\zeta)$, and $g^{\#}(\zeta) \leq g^{\#}(0)=k A+1$. where

$g^{\#}(\zeta)=\frac{\left|g^{\prime}(\zeta)\right|}{1+|g(\zeta)|^{2}}$ is said to be Spherical derivative of $g$.

Lemma 2 [5]. Let $f(z)$ be nonconstant meromorphic function in $C$

$$
\begin{gathered}
\Theta(a, f)=1-\varlimsup_{r \rightarrow \infty} \frac{\bar{N}\left(r, \frac{1}{f-a}\right)}{T(r, f)},(a \neq \infty), \\
\Theta(\infty, f)=1-\varlimsup_{r \rightarrow \infty} \frac{\bar{N}(r, f)}{T(r, f)}
\end{gathered}
$$

then $\sum_{a \in C}[\Theta(a, f)]+\Theta(\infty, f) \leq 2$.

\section{Proof of Theorem}

Suppose that $F$ be not normal in $\Delta$, then by Lemma 1 we get that there exists $f_{n} \in F, z_{n} \in \Delta$ and $\rho_{n} \rightarrow 0^{+}$ such that $g_{n}(\zeta)=\frac{f_{n}\left(z_{n}+\rho_{n} \zeta\right)}{\rho_{n}^{k}}$ converges locally and uniformly to a noncontant meromorphic function $g(\zeta)$ [6], and $g \#(\zeta) \leq g \#(0)=k c+1$. We claim that the following conclusions hold.

1) zeros of $g(\zeta)$ have multiplicity $k$ at least, and $g(\zeta)=0 \Rightarrow\left|g^{(k)}(\zeta)\right| \leq c$;

2) $g^{(k)}(\zeta) \neq a, g^{(k)}(\zeta) \neq b$;

In fact, suppose that there exists $\xi_{0} \in \Delta$, such that $g\left(\zeta_{0}\right)=0$, there exists $\zeta_{n} \rightarrow \zeta_{0}$,

$g_{n}\left(\zeta_{n}\right)=\frac{f_{n}\left(z_{n}+\rho_{n} \zeta_{n}\right)}{\rho_{n}^{k}}=0$ for sufficiently large $n$,

Hence $f_{n}\left(z_{n}+\rho_{n} \zeta_{n}\right)=0$.

Therefore, the following conclusions is obviously

$$
f_{n}^{(j)}\left(z_{n}+\rho_{n} \zeta_{n}\right)=0,(j=1,2,3, \cdots, k-1)
$$

and $\left|f_{n}^{(k)}\left(z_{n}+\rho_{n} \zeta_{n}\right)\right| \leq c$,

Hence

$g_{n}^{(j)}\left(\zeta_{n}\right)=\rho_{n}^{j-k} f_{n}^{(j)}\left(z_{n}+\rho_{n} \zeta_{n}\right)=0,(j=1,2,3, \cdots, k-1)$

and $\left|g_{n}^{(k)}\left(\zeta_{n}\right)\right| \leq c$,

$$
\text { So } g^{(j)}\left(\zeta_{0}\right)=\lim _{n \rightarrow \infty} g_{n}^{(j)}\left(\zeta_{n}\right)=0,(j=1,2,3, \cdots, k-1)
$$

and $\left|g^{(k)}\left(\zeta_{0}\right)\right| \leq c$,

Hence, zeros of $g(\zeta)$ have multiplicity $k$ at least, and $g(\zeta)=0 \Rightarrow\left|g^{(k)}(\zeta)\right| \leq c$,

Therefore, conclusion (1) is hold

In what follow, we complete the proof of the claim 2): Suppose that there exists $\zeta_{0}$

Such that $g^{(k)}\left(\zeta_{0}\right)=a$, by Hurwitz theorem, there exists $\zeta_{n} \rightarrow \zeta_{0}$ such that $g_{n}^{(k)}\left(\zeta_{n}\right)=f_{n}^{(k)}\left(z_{n}+\rho_{n} \zeta_{n}\right)=a$ for sufficiently large $n$.

By conditions:

$$
\begin{gathered}
\bar{E}_{f^{(k)}}(S) \subseteq \bar{E}_{f}(S), \\
f_{n}\left(z_{n}+\rho_{n} \zeta_{n}\right)=a(\text { or } b),
\end{gathered}
$$

Hence

$$
g\left(\zeta_{0}\right)=\lim _{n \rightarrow \infty} g_{n}\left(\zeta_{n}\right)=\lim _{n \rightarrow \infty} \frac{f_{n}\left(z_{n}+\rho_{n} \zeta_{n}\right)}{\rho_{n}^{k}}=\infty,
$$

this is a contradictions for $g^{(k)}\left(\zeta_{0}\right)=a$.

If $g^{(k)}(\zeta) \equiv a$, then $g(\zeta)$ is polynomial of degree $k$.

Because, zeros of $g(\zeta)$ have multiplicity $k$ at least, then $g(\zeta)=\frac{a}{k !}\left(\zeta-\zeta_{0}\right)^{k}$

and $g^{\prime}(\zeta)=\frac{a}{(k-1) !}\left(\zeta-\zeta_{0}\right)^{k-1}$

Obviously

$$
g^{\#}(0)=\frac{\left|\frac{a}{(k-1) !}\left(-\zeta_{0}\right)^{k-1}\right|}{1+\left|\frac{a}{k !}\left(-\zeta_{0}\right)^{k}\right|} \leq\left\{\begin{array}{l}
\frac{k}{2},\left|\zeta_{0}\right|>1 \\
\frac{|a|}{(k-1) !},\left|\zeta_{0}\right| \leq 1
\end{array}\right.
$$

Because $\left|g^{(k)}\left(\zeta_{0}\right)\right|=|a| \leq c$, then 
$\frac{|a|}{(k-1) !} \leq \frac{c}{(k-1) !}<k c+1$, Suppose $c \geq 1$ is not general, therefore $\frac{k}{2}<k c+1$, hence $|g \#(0)|<k c+1$, this is a concontradictions for conditions of $g(\zeta)$. Hence $g^{(k)}(\zeta) \neq a$. Similar to prove $g^{(k)}(\zeta) \neq b$. Therefore

$$
\begin{aligned}
& \Theta\left(a, g^{(k)}\right)=1-\varlimsup_{r \rightarrow \infty} \frac{\bar{N}\left(r, \frac{1}{g^{(k)}-a}\right)}{T\left(r, g^{(k)}\right)}=1 \text { and } \\
& \Theta\left(b, g^{(k)}\right)=1-\varlimsup_{r \rightarrow \infty} \frac{\bar{N}\left(r, \frac{1}{g^{(k)}-b}\right)}{T\left(r, g^{(k)}\right)}=1
\end{aligned}
$$

Since

$$
\begin{aligned}
& T\left(r, g^{(k)}\right) \geq N\left(r, g^{(k)}\right) \\
& =k \bar{N}(r, g)+N(r, g) \geq(k+1) \bar{N}(r, g)
\end{aligned}
$$

So

$$
\Theta\left(\infty, g^{(k)}\right)=1-\varlimsup_{r \rightarrow \infty} \frac{\bar{N}\left(r, g^{(k)}\right)}{T(r, g(k))} \geq 1-\frac{1}{k+1}=\frac{k}{k+1}
$$

Therefore

$$
\Theta\left(a, g^{(k)}\right)+\Theta\left(b, g^{(k)}\right)+\Theta\left(\infty, g^{(k)}\right) \geq 2+\frac{k}{k+1}>2
$$

this is a contradictions for Lemma 2. The proof of Theorem is completed.

\section{References}

[1] W.-H. Zhang, "The Normality of Meromorphic Functions Concerning One-Way Sharing Set," Journal of Nanhua University, Vol. 18, 2004, pp. 36-38.

[2] W.-H. Zhang, "The Normality of Meromorphic Functions," Journal of Nanhua University, Vol. 12, No. 6, 2004, pp. 709-711.

[3] F.-J. Lv and J.-T. Li, "Normal Families Related to Shared Sets," Journal of Chongqing University: English Edition, Vol. 7, No. 2, 2008, pp. 155-157.

[4] X. C. Pang and L. Zalcman, "Normal Families and Shared Values," Bulletin of London Mathematical Society, Vol. 32, No. 3, 2000, pp. 325-331. doi:10.1112/S002460939900644X

[5] W. K. Hayman, "Meromorphic Function," Oxford University Press, London, 1964.

[6] J. Clunie and W. Hayman, "The Spherical Derivative of Integral and Mer-Functions," Commentarii Mathematici Helvetici, Vol. 40, 1966, pp. 117-148. doi:10.1007/BF02564366 